\title{
Acetazolamide Stress SPECT Test on Cerebral Blood Flow in Patients with Depressive Disorder
}

\author{
Yi Xu*, Jian Gong, Hao Xu \\ Department of Clinical Psychology, The First Affiliated Hospital of Jinan University, Guangzhou, China
}

Email address:

drxuyi@sina.com (Yi Xu)

${ }^{*}$ Corresponding author

To cite this article:

Yi Xu, Jian Gong, Hao Xu. Acetazolamide Stress SPECT Test on Cerebral Blood Flow in Patients with Depressive Disorder. Advances in Applied Physiology. Vol. 1, No. 3, 2016, pp. 38-42. doi: 10.11648/j.aap.20160103.11

Received: July 3, 2016; Accepted: August 25, 2016; Published: October 28, 2016

\begin{abstract}
The regional cerebral blood flow (rCBF) and the cerebral vasoreactivity of the patients with depressive disorder were observed by SPECT and acetazolamide stress SPECT test respectively. Eighteen unmedicated depressed patients and nineteen healthy control subjects were included. Baseline SPECT was performed in both patients and control subjects. Acetazolamide stress SPECT test was performed two days later in patients two hours after taking $2 \mathrm{~g}$ acetazolamide orally. It was found that there was significant difference between the untreated depressed patients and the controls, because there was relatively reduced perfusion in frontal lobe, temporal lobe, left parietal lobe and right basal ganglia in depressed patients. After acetazolamide stress SPECT test, the perfusion of decreased regions in baseline step was increased. No potential ischemic lesion was found. The perfusion of some specific regions of the depressed patients decreased. Acetazolamide stress SPECT test could not reveal silent brain ischemia in the depressed patients. The perfusion of decreased regions in the baseline imaging was increased in acetazolamide stress SPECT test.
\end{abstract}

Keywords: Depression, Regional Cerebral Blood Flow, SPECT, Acetazolamide

\section{Introduction}

According to some clinical researches in recent years, the patients with depressive disorder might have asymmetry cerebral artery dysfunction $[1,2]$ and abnormality of $\mathrm{rCBF}[3$, 4]. However the clinical significance and the mechanism was not clear. Its role and meaning in the occurrence and the development of the depressive disorder should be further investigated. Here, rCBF of un-medicated patients was analyzed semi-quantitively using SPECT combined with Acetazolamide Stress Test (diamox test). The adjustment ability of the cerebral blood vessels of the patients in the region with decreased blood flow was observed and also whether there is potential ischemic lesion was studied.

\section{Objects and Methods}

\subsection{Depression Group}

18 patients with depressive disorder were hospitalized from Oct. 2010 to Mar. 2012 in the psychiatric ward of The First
Affiliated Hospital of Jinan University, among which 8 patients were male while 10 patients were female with the age ranging from 20 to $61(43.26 \pm 10.27)$. All of them were right-handed. Their outbreaks conformed to the diagnosis standard of depressive episode described in DSM-IV. The episode lasted from 20 to 90 days, with no mental disorder drug intake or drug withdrawal more than 7 days. The scores of 24 items from the Hamilton Depression Scale (HAMD) were above 20. The scores of 14 items from the Hamilton Anxiety Scale (HAMA) were above 7. The patients with depressive disorder but also with other physical diseases, brain organic diseases or other mental disorder were excluded. The patients with the complications which likely led to brain artery organic change were excluded as well. That complications included hypertension, hyperlipemia, diabetes mellitus, coronary disease and strokes.

\subsection{Normal Controls}

There was in total 19 people in the normal control group who participated in the physical examination in The First 
Affiliated Hospital of Jinan University from Mar. 2010 to Dec. 2012, among which 8 people were male and 11 people were female with the age ranging from 21 to 64 years old $(42.61 \pm 10.88)$. They were all right-handed. There was no significant difference of the average age and sex proportion between the patient group and the control group.

\subsection{Assessment by HAMD and HAMA}

Once the patients were hospitalized, medical history was enquired in detail and physical examination was performed on them. Biomedical test, ECG and brain CT (Hispeed, GE Medical system) was performed within three days of hospitalization. The patients were selected afterwards. In order to assess if the depression or anxiety level of the patients fulfilled the diagnosis criteria, the patient group was assessed by HAMD with 24 items and HAMA with 14 items within two days of hospitalization before any antidepressant treatment. The assessment of all the scales of the patients was made by the same doctor from our research group to make sure all results were scored under same criteria.

\subsection{Brain SPECT and Acetazolamide Stress Test}

Brian SPECT and acetazolamide stress test were performed on the patient group within three days of hospitalization before any antidepressant treatment. For the control group only brain SPECT was performed.

(1) Brain SPECT investigation: $400 \mathrm{mg}$ of potassium perchlorate was taken orally to block the uptake of ${ }^{99} \mathrm{Tc}^{\mathrm{m}} \mathrm{O}_{4}$ by choroid plexus. After $30 \mathrm{~min}, 740 \sim 925 \mathrm{MBq}$ of Technetium ${ }^{99 m}$-ethylcysteinated $\left({ }^{99} \mathrm{Tc}^{\mathrm{m}}\right.$-ECD) was injected via cubital veins, which was bought from Guangzhou Medical Isotope Service Centre of China Nuclear Science Institute. It was proved to be qualified by routine quality control after labeling and its radiochemical purity was above $95 \%$ determined by paper chromatography. Data collection: Tests started 30 min after the injection. The testee lied in a supine position on the scintibed with the head kept still, examined by the multifunctional SPECT instrument (GE Apex SPX HELIX) with dual probes. This instrument had ultrahigh resolution fan shape collimator, whose collecting matrix was $128 \times 128$ and amplification factor was 1.0. Totally sixty frames were collected step-by-step with one frame every six degree. Each frame took 25 30 sec. Hanning filtering was used for rebuilding with attenuation correction coefficient of 0.11 . The cross sectional images paralleling to OM line, slice-images of frontal plane and sagittal plane were obtained.

(2) Acetazolamide Stress Test: The patients orally took $2 \mathrm{~g}$ of acetazolamide (Jiansu Chenpai Pharmaceutical Company Limited) $48 \mathrm{~h}$ after brain SPECT test. After 2 $\mathrm{h}$ these patients were tested again by SPECT test.

(3) SPECT Image Analysis: The semi-quantitative analysis of $\mathrm{rCBF}$ in cerebral cortex used dedicated software (Semi-Quantitative Brain Following Slicing) on X-Pert (V5.13) workstation. For each subject, the SPECT image that best passed through the inferior lobes was selected. Cortex was outlined by a ring extending from the outer brain edge inward according each brain's diameter toward the brain centroid. The cortical ring included samples of various cerebral cortex from frontal, temporal, parietal, and occipital lobes. The mean cortical value averaged across the cortical ring regions in selected slice and the next slice was used as the index measure of relative cerebral cortex perfusion. The basal ganglia and cerebellum was outlined at the slices which best passed through the middle of the basal ganglia and cerebellum, and the mean SPECT value from these region were used as the relative measure of basal ganglia and cerebellar rCBF.

Because these SPECT measures are relative blood flow units, it is necessary to normalize these analysis regions by adjusting the image intensity according to the cerebellar mean value. The mean cerebral cortex and basal ganglia SPECT values were compared with the mean cerebellum SPECT values by computing the ratio of the index region mean image value, divided by the reference region mean value $\times 100$. Thus, regional measures of these brain structures from these normalized images have a mean value that is a direct percentage of the cerebellar mean value.

\subsection{Statistical Method}

SPSS 10.0 statistical package was used. All tests were bilateral hypothesis tests with level of significant (a) equaled to 0.05 . All the measured data were analyzed with unitization mean $\mathrm{t}$ test.

\section{Result}

\subsection{Result of Two Group SPECT Tests}

Normal group SPECT images: the radioactive distribution of blood flow of each pair of cerebral function regions was approximately even and bilaterally symmetric. The SPECT image of a normal human brain was shown in Fig. 1A.

The comparison of semi-quantitative score of $\mathrm{rCBF}$ between the depression group and the control group was shown in Table 1. For the depressive group, the rCBF of left frontal lobe and left temporal lobe decreased significantly $(p<$ $0.01)$. The rCBF of right frontal lobe, right temporal lobe, left parietal lobe and right basal ganglia also decreased $(p<0.05)$. At the same time, the low perfusion of $\mathrm{rCBF}$ of frontal lobe, temporal lobe and parietal lobe was asymmetric. The low perfusion of rCBF of the left side was obvious. However the perfusion of $\mathrm{rCBF}$ of right basal ganglia was lower than that of the left side. 
Table 1. Comparison of semi-quantitative score of $r C B F$ between depressive group and control group.

\begin{tabular}{lllll}
\hline \multirow{2}{*}{ Site } & Right & & Left & Depression group \\
\cline { 2 - 5 } & Control group & Depression group & Control group & $50.24 \pm 3.11^{233)}$ \\
\hline Frontal lobe & $63.01 \pm 7.46$ & $58.10 \pm 3.32^{1)}$ & $61.06 \pm 7.73$ & $53.17 \pm 4.16^{233)}$ \\
Temporal lobe & $62.80 \pm 7.67$ & $58.34 \pm 4.24^{1)}$ & $62.32 \pm 8.13$ & $58.22 \pm 4.17$ \\
Parietal lobe & $65.27 \pm 6.92$ & $62.68 \pm 3.36$ & $63.17 \pm 7.29$ & $67.65 \pm 6.12$ \\
Occipital lobe & $70.59 \pm 7.40$ & $67.90 \pm 6.90$ & $71.92 \pm 6.90$ & $61.50 \pm 4.90$ \\
Basal ganglia & $64.99 \pm 6.94$ & $60.51 \pm 4.67$ & $64.11 \pm 5.93$ & \\
\hline
\end{tabular}

1) Compared with control group, $p<0.05$ by $t$ test.

2) Compared with control group, $p<0.01$ by $t$ test.

3) Compared with right side in depressive group, $p<0.05$ by $t$ test.

\subsection{Result of Acetazolamide Stress SPECT Test of Depression Group}

Five depressive patients whose SPECT test results were normal still showed normal cerebral blood flow after the intake of acetazolamide. The $\mathrm{rCBF}$ of thirteen patients whose $\mathrm{rCBF}$ was lower originally became normal after the intake of acetazolamide shown in Fig. 1B and C.
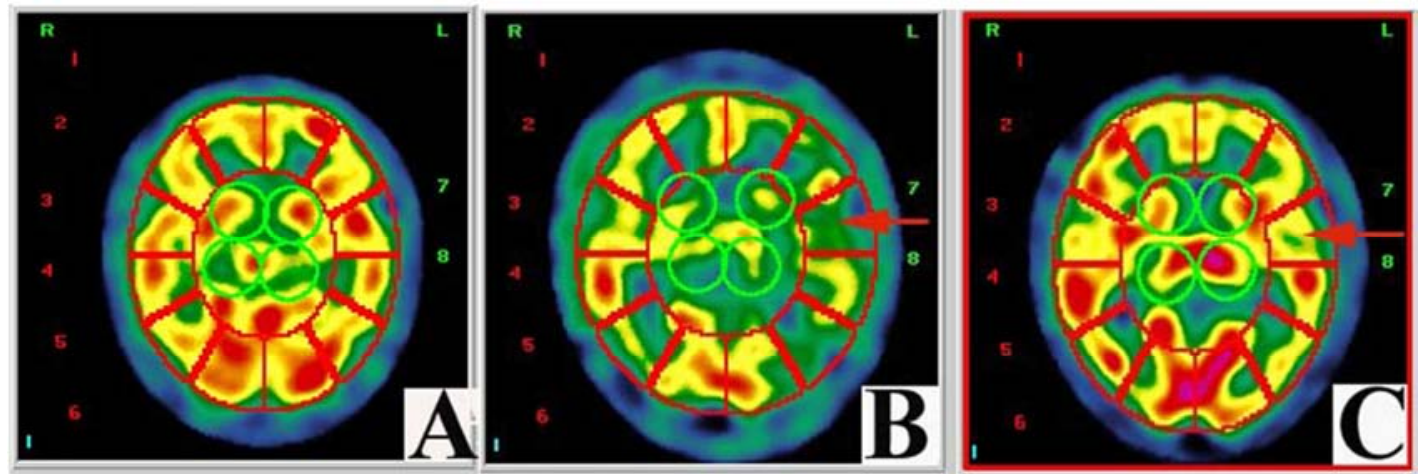

Figure 1. $r C B F$ SPECT results in a patient with depressive disorder.

(A) rCBF SPECT result in a control subject;

(B) left temporal hypoperfusion before diamox test;

(C) increased left temporal hypoperfusion after diamox test.

Semi-quantitative Analysis: As shown in table 2, after the intake of acetazolamide, the rCBF remained unchanged for those regions such as right parietal lobe, bilateral occipital lobe, left basal ganglia whose $\mathrm{rBCF}$ was normal originally. The rCBF of those regions including bilateral frontal lobe, bilateral temporal lobe, left parietal lobe and right basal ganglia with decreased $\mathrm{rCBF}$ originally improved significantly after the intake of acetazolamide $(p<0.05)$. There was significant difference between the rCBF of two sides but now there was not $(p>0.05)$.

Table 2. Comparison of semi-quantitative score of $r$ CBF before and after acetazolamide SPECT in 18 patients with depressive disorder.

\begin{tabular}{lllll}
\hline \multirow{2}{*}{ Site } & Right & & Left & After diamox test \\
\cline { 2 - 5 } & Before diamox test & After diamox test & Before diamox test & $58.48 \pm 6.29$ \\
\hline Frontal lobe & $58.10 \pm 3.32$ & $61.25 \pm 3.69^{1)}$ & $50.24 \pm 3.11$ & $56.66 \pm 4.46$ \\
Temporal lobe & $58.34 \pm 4.24$ & $61.66 \pm 5.25^{1)}$ & $53.17 \pm 4.16$ & $61.27 \pm 3.57$ \\
Pariental lobe & $62.68 \pm 3.36$ & $60.09 \pm 4.00$ & $58.22 \pm 4.17$ & $69.96 \pm 5.13$ \\
Occipital lobe & $67.90 \pm 6.90$ & $68.58 \pm 5.43$ & $67.65 \pm 6.12$ & $60.17 \pm 4.79$ \\
Basal ganglia & $60.51 \pm 4.67$ & $63.76 \pm 4.71$ & $61.50 \pm 4.90$ & \\
\hline
\end{tabular}

1) Compared with before diamox test, $p<0.05$ by $t$ test.

After the intake of the medicine, the $\mathrm{rCBF}$ of depression group was compared with that of the control group. The rCBF of all regions except left temporal lobe restored to normal.

\section{Discussion}

Recent investigations on the cerebral blood flow of the depressive patients showed that the $\mathrm{rCBF}$ of some patients decreases, namely the rCBF low perfusion in frontal lobe and temporal lobe $[3,5]$. The perfusion decrease was found also in limbic system [6]. It was found that the whole brain or left lateral cerebral blood flow perfusion changed, but most people agreed in low perfusion in frontal lobe and temporal lobe [7-10].

It could be observed by eye that, in thirteen among eighteen patients, the rCBF low perfusion existed in various regions such as frontal lobe, temporal lobe, parietal lobe, occipital 
lobe and basal ganglia. After semi-quantitative analysis of SPECT of depressive group and control group, it was found that compared with the control group, the rCBF perfusion decreased in frontal lobe, temporal lobe, left parietal lobe and right basal ganglia of the depression group. rCBF low perfusion was asymmetric with that of the left side more obvious, the difference was significant which meant that $\mathrm{rCBF}$ had some relationship with the depressive disorder [10]. Although in most cases shown by SPECT tests that there was intracranial ischemia in people with depressive disorder, there were researches indicating the normal intracranial perfusion. In five cases there was no change of rCBF perfusion observed by naked eyes. It should be further studied if in those cases there was latent ischemic lesions which was not observed by base state SPECT. Therefore we used Acetazolamide stress SPECT test to investigate it. Acetazolamide stress SPECT test was proven to be able to find intracranial potential focal ischemia in ischemic cerebrovascular disease [11]. Acetazolamide is a potent, reversible carbonic anhydrase inhibitor. Acetazolamide makes normal blood vessels to dilate thus increasing blood perfusion [12]. However lesion vessels did not expand or just a little and blood perfusion could not increase or just a little. Therefore many foreign scholars adopted intravenous injection of acetazolamide to improve the positive detection rate of ischemic cerebrovascular disease. But acetazolamide for intravenous injection cannot be manufactured in China and needs to be imported from abroad which makes it very expensive and limits its clinical application. Acetazolamide tablets are cheap and easy to be obtained, have the identical molecular structure as that of the injection formulation. The difference between them are the route of administration, onset time, efficacy peak time and action time. Studies indicates that acetazolamide oral absorption was very good [13, 14]. After about two hours plasma concentration reached the peak. The $\mathrm{pH}$ value of the blood decreased obviously after the intake of the medicine. PCO2 was significantly higher than that before the medication, approaching the effects of intravenous injection.

Five patients from the depressive group with normal SPECT test result did not have any new ischemia or other abnormal cerebral blood flow after oral intake of acetazolamide. On the contrary, the low perfusion situation of all the patients with decreased rCBF improved obviously and basically restored to normal after the oral intake of acetazolamide. The phenomenon of asymmetry of rCBF perfusion between the left and the right side disappeared completely, which was absolutely different from the result of acetazolamide stress test in ischemic cerebrovascular disease indicating that the patients didn't have any cerebral vascular disease and vascular reactivity was good. The original ischemia regions restored to normal blood flow perfusion status because of the vascular expansion function of acetazolamide. This is consistent with current theory that the depressive disorder is a functional disease and the patients have no cerebrovascular disease [1]. Osihi performed brain SPECT and intravenous acetazolamide brain SPECT tests on patients with chronic alcoholism [15]. It was found that the cerebral blood flow perfusion decreased for base state SPECT while it increased obviously after intravenous injection of acetazolamide, which showed that the decrease of cerebral blood flow perfusion of the patients with chronic alcoholism was not due to the pathological changes of the vessel itself but due to the decrease of brain metabolism.

The pathogenesis of depressive disorder is complicated. Up till now it is not clear whether the decrease of rCBF in the patients with depressive disorder is the cause of this disease or the result caused by the disease. Bonne thought that the decrease of $\mathrm{rCBF}$ of the patients was a status signal and $\mathrm{rCBF}$ perfusion restored with the improvement of the disease [16]. rCBF perfusion increased after the oral intake of acetazolamide. It still needs further investigation to decide if the above phenomenon is beneficial to the recovery of the disease or to the identification of depressive disorder and depressive disorder with occult-phase ischemic cerebrovascular disease.

\section{References}

[1] Neu, P., P. Schlattmann, A. Schilling, and A. Hartmann, Cerebrovascular reactivity in major depression: a pilot study. Psychosom Med, 2004, 66 (1): 6-8.

[2] Devantier, T. A., B. L. Norgaard, M. K. Poulsen, E. Garde, K. A. Ovrehus, M. Marwan, S. Achenbach, D. Dey, L. H. Sorensen, and P. Videbech, White Matter Lesions, Carotid and Coronary Atherosclerosis in Late-Onset Depression and Healthy Controls. Psychosomatics, 2016.

[3] Kaichi, Y., G. Okada, M. Takamura, S. Toki, Y. Akiyama, T. Higaki, Y. Matsubara, Y. Okamoto, S. Yamawaki, and K. Awai, Changes in the regional cerebral blood flow detected by arterial spin labeling after 6-week escitalopram treatment for major depressive disorder. J Affect Disord, 2016, 194: 135-43.

[4] Ota, M., T. Noda, N. Sato, K. Hattori, T. Teraishi, H. Hori, A. Nagashima, K. Shimoji, T. Higuchi, and H. Kunugi, Characteristic distributions of regional cerebral blood flow changes in major depressive disorder patients: a pseudo-continuous arterial spin labeling (pCASL) study. J Affect Disord, 2014, 165: 59-63.

[5] Chen, Z. Q., M. Y. Du, Y. J. Zhao, X. Q. Huang, J. Li, S. Lui, J. M. Hu, H. Q. Sun, J. Liu, G. J. Kemp, and Q. Y. Gong, Voxel-wise meta-analyses of brain blood flow and local synchrony abnormalities in medication-free patients with major depressive disorder. J Psychiatry Neurosci, 2015, 40 (6): 401-11.

[6] Gonul, A. S., M. Kula, A. G. Bilgin, A. Tutus, and A. Oguz, The regional cerebral blood flow changes in major depressive disorder with and without psychotic features. Prog Neuropsychopharmacol Biol Psychiatry, 2004, 28 (6): 1015-21.

[7] Vangu, M. D., J. D. Esser, I. H. Boyd, and M. Berk, Effects of electroconvulsive therapy on regional cerebral blood flow measured by 99mtechnetium HMPAO SPECT. Prog Neuropsychopharmacol Biol Psychiatry, 2003, 27 (1): 15-9.

[8] Bhardwaj, R., S. Chakrabarti, B. R. Mittal, and P. Sharan, A single photon emission computerized tomography (SPECT) study of regional cerebral blood flow in bipolar disorder. World J Biol Psychiatry, 2010, 11 (2 Pt 2): 334-43. 
[9] Kawakatsu, S. and A. Komatani, [Xe-133 inhalation single photon emission computerized tomography in manic-depressive illness]. Nihon Rinsho, 1994, 52 (5): 1180-4.

[10] 1Hosokawa, T., T. Momose, and K. Kasai, Brain glucose metabolism difference between bipolar and unipolar mood disorders in depressed and euthymic states. Prog Neuropsychopharmacol Biol Psychiatry, 2009, 33 (2): 243-50.

[11] Vagal, A. S., J. L. Leach, M. Fernandez-Ulloa, and M. Zuccarello, The acetazolamide challenge: techniques and applications in the evaluation of chronic cerebral ischemia. AJNR Am J Neuroradiol, 2009, 30 (5): 876-84.

[12] Kojima, T., S. Mizumura, S. I. Kumita, H. Nakajo, T. Kumazaki, T. Kitamura, K. Fukino, and A. Teramoto, Regional cerebral blood flow and vascular reserve in neuronal migration disorder assessed by Tc-99m hexamethylpropylene amineoxime SPECT with acetazolamide challenge. Clin Nucl Med, 2000, 25 (9): 749-50.
[13] Saeed, A., M. Al-Rashida, M. Hamayoun, A. Mumtaz, and J. Iqbal, Carbonic anhydrase inhibition by 1-aroyl-3-(4-aminosulfonylphenyl) thioureas. J Enzyme Inhib Med Chem, 2014, 29(6):901-5.

[14] Granero, G. E., M. R. Longhi, C. Becker, H. E. Junginger, S. Kopp, K. K. Midha, V. P. Shah, S. Stavchansky, J. B. Dressman, and D. M. Barends, Biowaiver monographs for immediate release solid oral dosage forms: acetazolamide. J Pharm Sci, 2008, 97 (9): 3691-9.

[15] Oishi, M., Y. Mochizuki, and T. Takasu, Cerebral blood flow and cerebrovascular response to acetazolamide in patients with chronic alcoholism. J Neurol Neurosurg Psychiatry, 1997, 63 (1): $100-2$.

[16] Bonne, O. and Y. Krausz, Pathophysiological significance of cerebral perfusion abnormalities in major depression-trait or state marker? Eur Neuropsychopharmacol, 1997, 7 (3): 225-33. 